\title{
Effects of a preparatory training protocol on the movement and body stability of handball players
}

\author{
N. Rađević a , S. Simović b, ${ }^{*}$, N. Ponorac c ${ }^{\text {, D. Drljačić d }}$
}

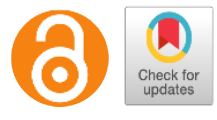

a Pan-European University Apeiron, Faculty of Sport Sciences, Banja Luka, the Republic of Srpska, Bosnia and Herzegovina

b University of Banja Luka, Faculty of Physical Education and Sport, Banja Luka, the Republic of Srpska, Bosnia and Herzegovina

c University of Banja Luka, Faculty of Medicine, Banja Luka, the Republic of Srpska, Bosnia and Herzegovina

d College of Sports and Health, Belgrade, Serbia, Sports Diagnostic Center Šabac, Šabac, Serbia

*Corresponding author Ph: 0038765580750 ; Email: slobodan.simovic@ffvs.unibl.org

DOI: https://doi.org/10.34256/ijpefs2146

Received: 03-10-2021, Revised: 08-11-2021; Accepted: 10-11-2021; Published: 16-11-2021

Abstract: Handball is a sport with a high risk of injury, The prevalence of injury is only obtainable through a thorough and comprehensive analysis. One of the most commonly used test batteries for interpreting the characteristics of an injury is the Functional Movement Screen $\left(\mathrm{FMS}^{\mathrm{TM}}\right)$. It makes possible to possible to identify movement limitations and asymmetries which are believed to impact injury risk in sports. The aim of this study is to use the $\mathrm{FMS}^{\mathrm{TM}}$ to determine whether an eight-week training protocol can predict and prevent injuries in handball. The study sample comprised the fifteen members of the Borac handball club youth team. The initial measurement showed that most players (80\%) had an overall score in the test battery ranging from 15 to 20 points. In addition, three players were found to have asymmetry. Only one of the three players had an overall score in the $\mathrm{FMS}^{\mathrm{TM}}$ of $\leq 14$. The participants scored the lowest in the initial measurement for Rotary Stability, followed by Deep Squat and Hurdle Step Left. They scored the highest in the Shoulder Mobility. After the implementation of the exercise protocol for improving body mobility and stability, the final measurements showed that all the participants had an overall score in the FMS ${ }^{\mathrm{TM}}$ of $>14$. The value of eta squared showed that training in between the two measurements had a significant impact. At the time of testing and protocol implementation no players sustained any injuries during matches or in training. This study confirmed that the $\mathrm{FMS}^{\mathrm{TM}}$ can be used to predict injuries in sports.

Keywords: Final Measurement, Functional Movement Screen, Injury, Initial Measurement, Mobility, Young Athletes

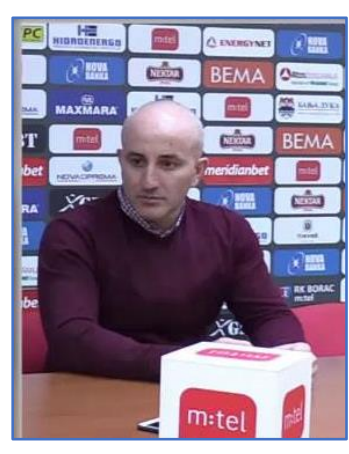

Nenad Rađević, Ph.D., graduated from the University of Banja Luka in 2004 at the Faculty of Physical Education and Sports. Since 2006, he has been working in the Ministry of Family, Youth, and Sports, Government of the Republic of Srpska. He received his master's degree from the Faculty of Physical Education and Sports in 2011 and completed his doctoral studies in 2018. He is a master of judo and a former member of the national judo team of Bosnia and Herzegovina. Since 2019, he has been working as an assistant professor at the Paneuropean University "Apeiron" Banja Luka, Faculty of Sports Sciences, on General
Anthropomotorics. For the last five years, he has been working as a fitness coach at the Handball Club "Borac" Banja Luka.

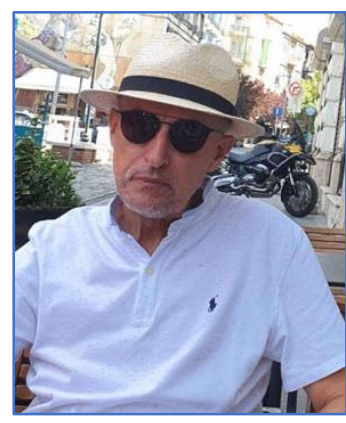

Slobodan Simović, Ph.D., is an associate professor at the Faculty of Physical Education and Sport, University of Banja Luka. He graduated from the department of basketball at the Faculty of Sports in Sarajevo and graduated management in sports in Belgrade, where he completed his postgraduate and doctoral studies. He teaches basketball and management in sports, which are also his fields of research. He is taking part in 
several national and international scientific projects. He was a professional basketball coach, leading several renowned teams from former Yugoslavia and Germany.

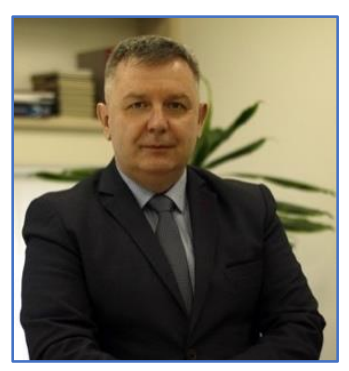

Nenad Ponorac, Ph.D., graduated from the University in Banja Luka, Faculty of Medicine in 2000. As of November 2000, he has been employed at the Faculty of Medicine, Department of Physiology. In 2007, he received a specialist degree in Sports Medicine, and in 2008 he successfully defended his Ph.D. thesis. As an Assistant Professor, he has taught Physiology and Sports Physiology at the Faculty of Medicine and Faculty of Physical Education and Sports, the University of Banjaluka since 2009, Associate Professor since 2014, and Full Professor since 2020. He has been a Visiting Professor at the Faculty of Physical Education and Sports, University of Nis, and Faculty of Medicine in Foča. He is also the author of four university textbooks. He is a reviewer and member of the editorial board at the medical journal Scripta Medica.

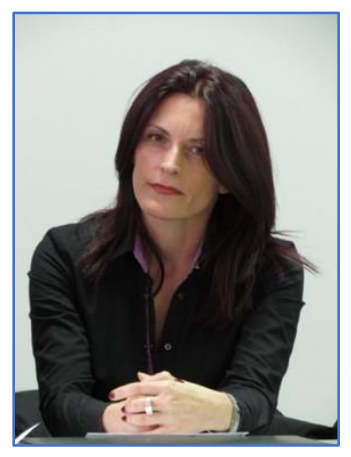

Dragana Drljača, Ph.D., is a professor of Kinesiology, Anthropomotorics, and Biomechanics of Sport at the College of Sports and Health, Belgrade. She also holds an appointment as a Head of the study program Vocational Physiotherapist and Project Team coordinator of the International Office of the same College. As an active member of the Association Sports Diagnostic Centre Sabac, she was engaged in several national and international projects focused on a healthy lifestyle and increasing physical activity, especially in children and youngsters. Currently, she participates in several Erasmus+ projects funded by the EU.

\section{Introduction}

Modern training technology has become an integral part of the daily routines set by conditioning coaches. A goal of both coaches and athletes is to improve physical abilities to achieve top results in sports. On one hand, the coach attempts to develop a high level of anaerobic and aerobic abilities, i.e. motor capacity, and on the other they have to make sure the athletes recuperate, to prevent injuries and to ensure proper movement performance. On that note, special attention needs to be paid to injury prevention, i.e. focusing experts on the improvement of the movement pattern itself [1-3].

Identifying risk of sports injuries and implementing appropriate preventive measures may help conditioning coaches reduce the occurrence of injuries, improve the athletes' performance in competitions, and minimize costs of healthcare for their players [4]. Prospective studies have identified several internal factors which increase the risk of sport injuries, including prior injuries, ligament laxity, limited movement range, low level of aerobic fitness or muscular strength, poor balance and lower level of prior physical activity or training [5-9]. Even though these factors are often studied separately, it is likely that there are multiple factors in the risk of injury. Sports medicine researchers need to determine how an athlete performs specific movement patterns in a particular sport, because it is believed that improper movement patterns and biomechanical faults often contribute to muscular and skeletal injuries in sports $[6,8,10-12]$.

Functional Movement Screen (FMS ${ }^{\mathrm{TM}}$ ) is one of the most commonly used test batteries for interpreting the quality of movements [13-18]. It is a non-invasive, inexpensive, quick, and easily implemented tool to assess multiple functional movement patterns of individuals to identify limitations of movement and asymmetry, which are believed to affect sports injury risk [19-23]. The rest is most commonly applied to athletes, but is also recommended for the general population [24]. Numerous studies explored the ability of $\mathrm{FMS}^{\mathrm{TM}}$ to serve as an injury prediction tool, but the conclusions of these studies are inconsistent, especially in the athlete population [7, 10-11, 22, 25-26]. Using the receiver operator characteristic (ROC) curve, several authors found that for most individuals, the overall score of $\leq 14$ out of the 21 possible points in the $\mathrm{FMS}^{\mathrm{TM}}$ represents a risk of injury $[22,25]$, while in other studies none of the results could predict an injury with any certainty $[16,27]$. A potential reason why the FMS $^{\mathrm{TM}}$ was unable to predict injuries for specific athletes is that $\mathrm{FMS}^{\mathrm{TM}}$ determines the total dysfunction of mobility, which can greatly differ from the mechanisms that cause sports injuries [4].

FMS $^{\mathrm{TM}}$ comprises a set of seven subtests [11, 17], which make up the functional basis of movement, which in turn allows for the evaluation and ranking of 
movements [28]. Each individual test is specific and uses stabilization movements, all of which require the individual to perform common basic movement patterns [28]. Stability and mobility are initial information which serve the coach for planning an adequate program for developing motor and functional capacity, but also for preserving and improving postural capacity. In this context, good mobility entails efficient performance of movements. It implies the interaction of specific body parts, i.e. multiple joints, when performing complex movements. When it comes to stability, it entails the ability to control one's body through strength, coordination, balance and movement quality [29]. There is static stability, which implies maintaining the appropriate body position and balance, and there is dynamic stability, which implies motion and movement control, i.e. the ability to counteract unwanted movements or forces. Looking at the human body, mobility should take place in the ankles, hips, thorax, shoulders and neck, while stability should come from the feet, knees, lumbar region and shoulder blades.

Handball is one of the most popular collective sports in the world, where team members pass the ball using their hands, trying to get it into the goal of the opposing team. This game features high tempo, sudden change of direction, jumps that result in powerful shots, frequent contact among the players, and repetitive injuries to knees and shoulders [30]. Therefore, it requires physical prowess and talent, as well as agility and tactics. According to the Olympic Committee, handball is an Olympic sport with a high risk of injury [31]. Handball players use functional movement patterns, just like other sports. Injuries can only be prevented by employing detailed and comprehensive analysis [32]. The most commonly used method for this analysis is FMS ${ }^{\mathrm{TM}}$.

Renan Hideki Higashi et al. (2015) found that the prevalence of injuries in youth handball players in a single season was $53.6 \%$ [33]. Further, other studies found that the injury frequency rate among elite male Brazilian handball players was 3.7 per 1000 hours of training and 20.3 per 1000 hours of match time [34] and 3.1 injuries per 1000 hours of training and 40.7 per 1000 hours of match time among female Brazilian players [35]. Again, other authors found that the number of injuries per 1000 hours of play was far larger, ranging from 104.5 [36] to 115.8 [37].

Furthermore, previous studies have shown that handball players have lower overall scores in the FMS ${ }^{\mathrm{TM}}$ than football players, which indicates that football players exhibit better functional movement patterns. The movements of football players were more symmetrical than those of handball players. Handball players had greater asymmetry of internal and external rotation of the shoulder. Results of the FMS ${ }^{\mathrm{TM}}$ lead to the assumption that handball players carry a greater risk of injury than football players [38]. The results of the $\mathrm{FMS}^{\mathrm{TM}}$ did not differ relative to the quality of handball players. In the study of Robert Slodownik et al. (2018) the average result of FMS ${ }^{\mathrm{TM}}$ of Polish players in the first-tier league was $15.5 \pm 1.9$, and in the second-tier league the result was $15.4 \pm 2.6$ points [39]. Since the results of the previous study were reevaluated after six months, it was concluded that the FMS $^{\text {TM }}$ can be a useful tool to recognize specific adaptations in the shoulder joints of handball players. Also, Amir Letafatkar et al. (2014) evaluated the FMS ${ }^{\mathrm{TM}}$ results for male and female football, basketball and handball players. The average grade for all athletes was 16.9 , but unfortunately the results were not broken down into categories of sport or sex [40].

Considering the fact that findings of previous studies did not consistent in terms of FMS ${ }^{\mathrm{TM}}$ as an injury prediction tool in athletes' population, the aim of this study was to use the FMSTM to determine the effects of implementing an eight-week introductory preparatory training protocol on the movement performance quality and body stability, as well as prediction and prevention of injuries among youth handball players of the Borac Banja Luka handball club.

\section{Materials and Methods}

The participant sample in this study comprised the junior team of the Borac Banja Luka handball club, a total of them $(n=15)$, aged $M=17.5 \pm 1.0$ years, mean height $M=187.8 \pm 6.8 \mathrm{~cm}(\operatorname{Min}=170.0 ;$ Max $=$ 197.0) and body mass at an average of $M=79.9 \pm 8.3$ $\mathrm{kg}$ ( Min = 70.0; Max = 95.0). The average Body Mass Index (BMI) of the young handball players was $M=$ $22.6 \pm 2.0($ Min = 21.0; $\operatorname{Max}=25.8)$.

The Borac Banja Luka handball club is the most successful handball club of Bosnia and Herzegovina of all time. The club was one of the rare handball clubs that was never relegated from the former first league of Yugoslavia throughout its existence, from 1957 to 1991. Players from Borac won six gold Olympic medals playing for Yugoslavia. The club won the European Champions Cup in the 1975/76 season and the European Handball Federation (EHF) cup in the 1990/1991 season. The club always paid special attention to their work on the youth system. The 
current generation of youth handball players in the 2020/2021 season won the Championship of the Republic of Srpska and the Premier League of Bosnia and Herzegovina in their age category. Youth handball players train on a daily basis and at least once a week they play official matches. In addition to the competition for youth categories at the level of the Republic of Srpska and Bosnia Herzegovina, they also compete in the first league of the Republic of Srpska for seniors.

The functionality of the locomotor apparatus of youth players was evaluated using the FMS ${ }^{T M}$ diagnostic test battery, developed by Gray Cook, Lee Burton and Barbara Hoogenboom [20]. This screening simplifies the evaluation of the basic movement patterns $[20,28]$ in relation to subtests: Deep Squat (DS), Hurdle Step (HS), In-Line Lunge (ILL), Shoulder Mobility (SM), Active Straight Leg Raise (ASLR), Trunk Stability Push - Pp (TSP), and Rotary Stability (RS). Measurement was done for both the initial and the final state. The two measurements were three months apart.

Three attempts were used for each subtest. Bilateral subtests were carried out on both sides, and both the left and the right side were evaluated, which is greatly significant in handball, considering player positions in the team, and the dominance of the left or right hand/foot. If there is a difference in values for the left and right side, the lower result is taken as final for the total sum of points. Once all three attempts are performed in each test, the best score is taken, valuated on a scale of 0 to 3 in the following way: $0=$ pain reported anywhere in the body; $1=$ unable to complete the movement pattern or unable to assume the position to perform the movement; 2 = able to complete the movement but must compensate in some way to perform the fundamental movement; $3=$ able to perform the movement correctly without any compensation; in accordance with standard expectations of movements associated with each test $[10-11,41]$.

There were $\sim 10$ s breaks between performances within each test, and a one-minute rest between the tests. The overall score of the FMS ${ }^{\mathrm{TM}}$ was calculated by adding up the grades from the individual tests.

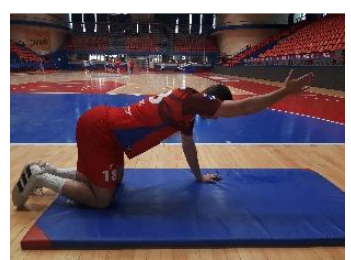

Hand anteflexion

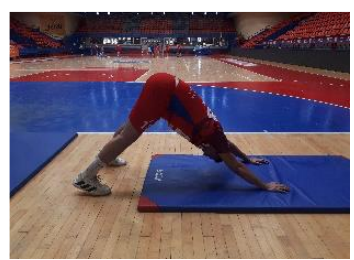

Hindu push-up

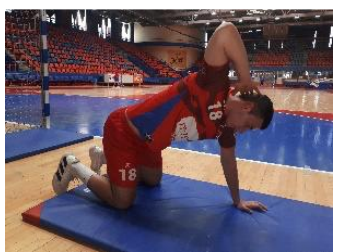

Opening the book

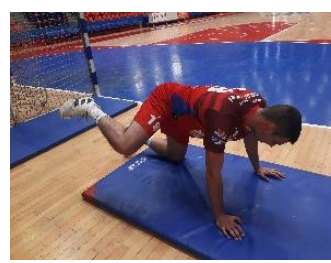

Hip abduction with

knee under 90

degrees

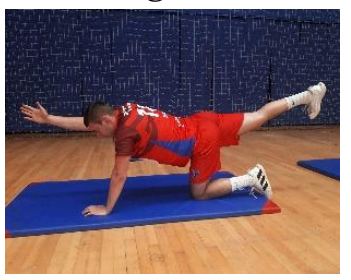

Hand anteflexion

and hip extension, same side

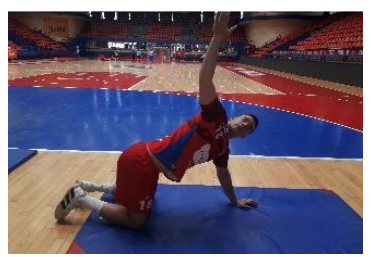

Shoulder opening with hand in extension

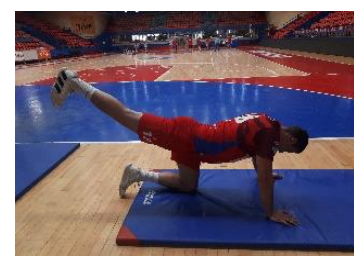

Hip extension

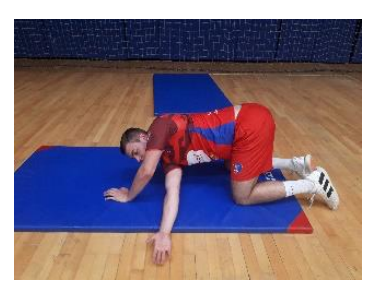

Needle

Needle

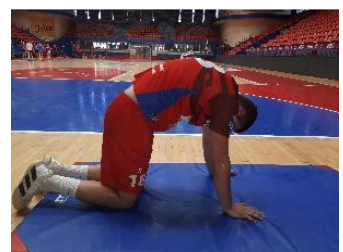

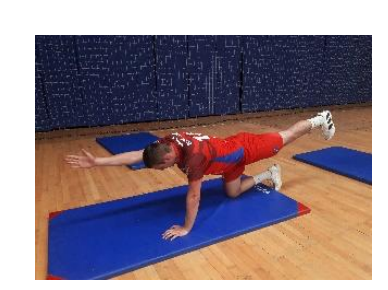

Superman

\section{Dog-cat}

Superman 


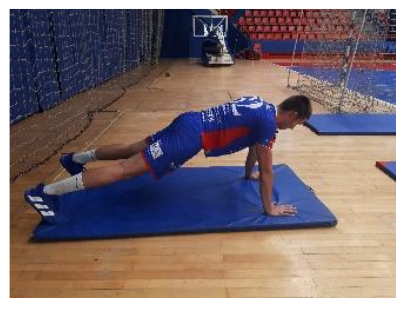

Front plank

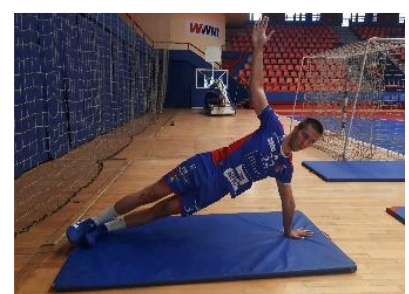

Side plank

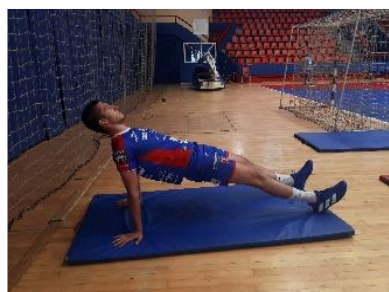

Back plank

Figure 2 Subprotocol of exercises intended for improving body stability.

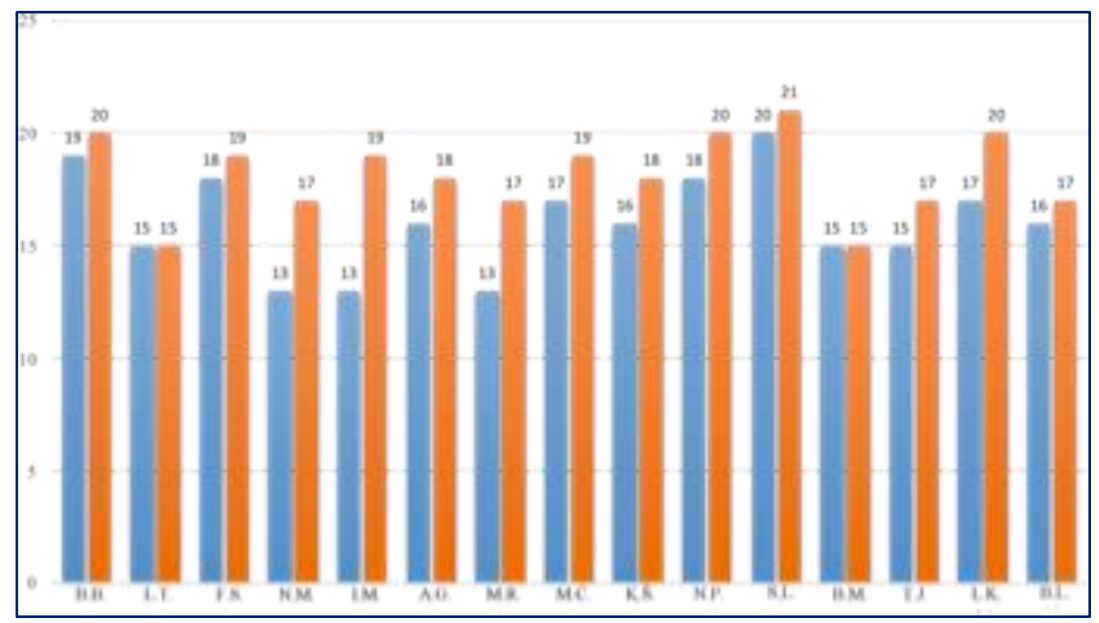

Figure 3 Differences in the overall score between the initial and final measurements in the study participants.

Table 1 Paired Samples Statistics and Correlations quality of movement performance and body stability of young handball players

\begin{tabular}{|c|c|c|c|c|c|c|}
\hline Variable & Measurement & $M$ & $S D$ & $S E$ & $r$ & $p$ \\
\hline \multirow{2}{*}{ DS } & Initial & 2.067 & 0.704 & 0.182 & \multirow{2}{*}{0.681} & \multirow{2}{*}{0.005} \\
\hline & Final & 2.600 & 0.507 & 0.131 & & \\
\hline \multirow{2}{*}{$H S_{\|}$} & Initial & 2.067 & 0.594 & 0.153 & \multirow{2}{*}{0.569} & \multirow{2}{*}{0.027} \\
\hline & Final & 2.600 & 0.507 & 0.131 & & \\
\hline \multirow[b]{2}{*}{$\mathrm{HS}_{\mathrm{rl}}$} & Initial & 2.133 & 0.516 & 0.133 & \multirow{2}{*}{0.491} & \multirow{2}{*}{0.063} \\
\hline & Final & 2.600 & 0.507 & 0.131 & & \\
\hline \multirow{2}{*}{$\mathrm{ILL}_{\|}$} & Initial & 2.400 & 0.507 & 0.131 & \multirow{2}{*}{0.218} & \multirow{2}{*}{0.435} \\
\hline & Final & 2.933 & 0.258 & 0.067 & & \\
\hline \multirow{2}{*}{ ILL $L_{r l}$} & Initial & 2.467 & 0.516 & 0.133 & \multirow{2}{*}{0.468} & \multirow{2}{*}{0.079} \\
\hline & Final & 2.800 & 0.414 & 0.107 & & \\
\hline \multirow[b]{2}{*}{$\mathrm{SM}_{\mathrm{ls}}$} & Initial & 2.733 & 0.458 & 0.118 & \multirow{2}{*}{0.650} & \multirow{2}{*}{0.009} \\
\hline & Final & 2.867 & 0.352 & 0.091 & & \\
\hline \multirow[b]{2}{*}{$S M_{r s}$} & Initial & 2.800 & 0.414 & 0.107 & \multirow{2}{*}{0.535} & \multirow[b]{2}{*}{0.040} \\
\hline & Final & 2.933 & 0.258 & 0.067 & & \\
\hline \multirow{2}{*}{$\mathrm{ASLR}_{\|}$} & Initial & 2.467 & 0.516 & 0.133 & \multirow{2}{*}{0.764} & \multirow{2}{*}{0.001} \\
\hline & Final & 2.600 & 0.507 & 0.131 & & \\
\hline \multirow{2}{*}{ ASLR } & Initial & 2.533 & 0.516 & 0.133 & \multirow{2}{*}{0.645} & \multirow{2}{*}{0.009} \\
\hline & Final & 2.733 & 0.458 & 0.119 & & \\
\hline \multirow{2}{*}{ TSP } & Initial & 2.600 & 0.507 & 0.131 & \multirow{2}{*}{0.739} & \multirow{2}{*}{0.002} \\
\hline & Final & 2.733 & 0.458 & 0.118 & & \\
\hline \multirow{2}{*}{ RS } & Initial & 1.867 & 0.516 & 0.133 & \multirow{2}{*}{0.607} & \multirow{2}{*}{0.016} \\
\hline & Final & 2.067 & 0.258 & 0.067 & & \\
\hline & Initial & 2.000 & 0.655 & 0.169 & & \\
\hline $\mathrm{RS}_{\mathrm{r}}$ & Final & 2.200 & 0.414 & 0.107 & 0.791 & 0.000 \\
\hline
\end{tabular}

$p<0.05$

Key: DS - Deep Squat; $\mathrm{HS}_{\|}$- Hurdle Step (left leg); $\mathrm{HS}_{\mathrm{rl}}$ - Hurdle step (right leg); ILL leg); ILL - In-line Lunge (right leg); SM $\mathrm{M}_{\mathrm{ls}}$ - Shoulder Mobility (left shoulder); SMrs - Shoulder Mobility (right shoulder); $A S L R_{\|}$- Active Straight-Leg Raise (left leg); ASLR $R_{r l}$ - Active Straight-Leg Raise (right leg); TSP - Trunk Stability Push Up; RS - Rotary Stability (left); RS $r_{r}$ Rotary Stability (right) 
Table 2 Paired samples test quality of movement performance and body stability of young handball players

\begin{tabular}{|c|c|c|c|c|c|c|c|c|c|}
\hline & \multicolumn{5}{|c|}{ Paired Differences } & \multirow{3}{*}{$t$} & \multirow{3}{*}{$d f$} & \multirow{3}{*}{$p$} & \multirow{3}{*}{$\varepsilon^{2}$} \\
\hline & \multirow[t]{2}{*}{$M$} & \multirow[t]{2}{*}{$S D$} & \multirow[t]{2}{*}{$S E$} & \multicolumn{2}{|c|}{$\begin{array}{l}\text { 95\% Confidence Interval } \\
\text { of the Difference }\end{array}$} & & & & \\
\hline & & & & Lower & Upper & & & & \\
\hline DS & -0.533 & 0.516 & 0.133 & -0.819 & -0.247 & -4.000 & 14 & 0.001 & 0.552 \\
\hline $\mathrm{HS}_{\|}$ & -0.533 & 0.516 & 0.133 & -0.819 & -0.247 & -4.000 & 14 & 0.001 & 0.552 \\
\hline $\mathrm{HS} \mathrm{rl}_{\mathrm{rl}}$ & -0.467 & 0.516 & 0.133 & -0.752 & -0.181 & -3.500 & 14 & 0.004 & 0.485 \\
\hline $\mathrm{ILL}_{\|}$ & -0.533 & 0.516 & 0.133 & -0.819 & -0.247 & -4.000 & 14 & 0.001 & 0.552 \\
\hline ILL $r 1$ & -0.333 & 0.488 & 0.126 & -0.604 & -0.063 & -2.646 & 14 & 0.019 & 0.350 \\
\hline$S M_{l s}$ & -0.133 & 0.352 & 0.091 & -0.328 & 0.063 & -1.468 & 14 & 0.164 & 0.142 \\
\hline SMrs & -0.133 & 0.352 & 0.091 & -0.328 & 0.062 & -1.468 & 14 & 0.164 & 0.142 \\
\hline $\mathrm{ASLR}_{\|}$ & -0.133 & 0.352 & 0.091 & -0.328 & 0.062 & -1.468 & 14 & 0.164 & 0.142 \\
\hline ASRLrl & -0.200 & 0.414 & 0.107 & -0.429 & 0.029 & -1.871 & 14 & 0.082 & 0.212 \\
\hline TSP & -0.133 & 0.352 & 0.091 & -0.328 & 0.062 & -1.468 & 14 & 0.164 & 0.142 \\
\hline $\mathrm{RS}_{\text {I }}$ & -0.200 & 0.414 & 0.107 & -0.429 & 0.029 & -1.871 & 14 & 0.082 & 0.212 \\
\hline $\mathrm{RS} r$ & -0.200 & 0.414 & 0.107 & -0.429 & 0.029 & -1.871 & 14 & 0.082 & 0.212 \\
\hline
\end{tabular}

Each participant could achieve a maximum of 21 points. The reliability of these evaluation protocols was determined with moderate to excellent level of congruence in trained assessors [42-43].

In the competition season, a specific training protocol was implemented (February-April 2021). It comprised two subprotocols which were implemented twice a week, in intervals of 10-15 minutes. The subprotocol of exercises directed at improving mobility of movements comprised 10 exercises (Figure 1), which were carried out in the introductory preparatory segment of the training session, following initial warmup, in the duration of 30 seconds for each exercise, i.e. for the left and right side.

The subprotocol of exercises intended for improving body stability comprised three exercises and four hold positions (Figure 2), which were also carried out in the introductory preparatory segment of the training session, following initial warm-up, in the duration of 45 seconds for each position, two sets each.

The data was statistically processed using the $T$-test of paired samples (repeated measurements), which ascertained whether there was a significant difference between the measured variables in the initial and final state. The amount of the impact of training, i.e. the obtained differences between the final and initial measurements was calculated using the Eta test.

\section{Results and Discussion}

Figure 3 displays the individual overall score of the initial and final measurements for the study participants. A statistically significant increase of the value of the Total Score from the initial $(M=16.067$, $S D=2.154)$ to the final measurement $(M=18.133$, $S D=1.807), \mathrm{t}(1)=-4.928, \mathrm{p}<0.000$ bidirectionally. The average decrease in the Total Score was 1.799, while the $95 \%$ confidence interval was in the range of -2.966 to -1.167 . The value of eta squared (2.152) indicates a high impact of the training.

Table 1 displays the score differences in descriptive statistics between the initial and final measurements and the correlative value for each individual test. The Paired Sample Test (Table 2) provided an evaluation of the effects of training on the test score.

- Deep Squad score from the initial $(M=2.067, S D$ $=0.704)$ to the final measurement $(M=2.600$, $S D=0.507), \mathrm{t}(14)=-4.000, \mathrm{p}<0.001$ bidirectionally. The average decrease in DS was 0.572 , while the $95 \%$ confidence interval was in the range of -0.819 to -0.247 . The value of eta squared was $\varepsilon^{2}=0.552$.

- Hurdle Step (left leg) (HS॥) score from the initial $(M=2.067, S D=0.704)$ to the final measurement $(M=2.600, S D=0.507), \mathrm{t}(14)=$ $4.000, \mathrm{p}<0.001$ bidirectionally. The average decrease in $\mathrm{HS}$ |I was -0.572 , while the $95 \%$ confidence interval was in the range of -0.819 to 0.247 . The value of eta squared was $\varepsilon^{2}=0.552$. 
- Hurdle Step (right leg) ( $\mathrm{HS}_{\mathrm{rl}}$ ) score from the initial $(M=2.133, S D=0.516)$ to the final measurement $(M=2.600, S D=0.507), \mathrm{t}(14)=$ $3.500, \mathrm{p}<0.004$ bidirectionally. The average decrease in $\mathrm{HS}_{\mathrm{rl}}$ was -0.544 , while the $95 \%$ confidence interval was in the range of -0.752 to 0.181 . The value of eta squared was $\varepsilon^{2}=0.485$.

- In-Line Lunge (left leg) (ILL॥) score from the initial $(M=2.400, S D=0.507)$ to the final measurement $(M=2.933, S D=0.258), \mathrm{t}(14)=$ $4.000, \mathrm{p}<0.001$ bidirectionally. The average decrease in IL $\|$ was -0.572 , while the $95 \%$ confidence interval was in the range of -0.819 to 0.247 . The value of eta squared was $\varepsilon^{2}=0.552$.

- In-Line Lunge (right leg) (ILLrl) score from the initial $(M=2.467, S D=0.516)$ to the final measurement $(M=2.800, S D=0.414), \mathrm{t}(14)=$ 2.646, $\mathrm{p}<0.019$ bidirectionally. The average decrease in $\mathrm{IL}_{r l}$ was -0.541 , while the $95 \%$ confidence interval was in the range of -0.604 to 0.063 . The value of eta squared was $\varepsilon^{2}=0.350$.

The results of the Paired Samples Test did not show a statistically significant increase of value, nor a high impact of training in:

- Active Straight-Leg Raise (right leg) (ASLR $R_{\mathrm{rl}}$ ) score from the initial $(M=2.533, S D=0.516)$ to the final measurement $(M=2.733, S D=0.458), \mathrm{t}(14)$ $=-1.871, \mathrm{p}<0.082$ bidirectionally. The average decrease in ASLR $r$ was -0.458 , while the $95 \%$ confidence interval was in the range of -0.429 to 0.029 . The value of eta squared was $\varepsilon^{2}=0.212$.

- Rotary Stability (left) (RSI) score from the initial $(M=1.867, S D=0.516)$ to the final measurement $(M=2.067, S D=0.258), \mathrm{t}(14)=$ $1.871, \mathrm{p}<0.082$ bidirectionally. The average decrease in RSI was -0.458 , while the $95 \%$ confidence interval was in the range of -0.429 to 0.029 . The value of eta squared was $\varepsilon^{2}=0.212$.

- Rotary Stability (right) (RSr) score from the initial $(M=2.000, S D=0.655)$ to the final measurement $(M=2.200, S D=0.414), \mathrm{t}(14)=$ $1.871, \mathrm{p}<0.082$ bidirectionally. The average decrease in RS, was -0.458 , while the interval of the $95 \%$ confidence was in the range of -0.429 to 0.029 . The value of eta squared was $\varepsilon^{2}=0.212$.

The results of the Paired Samples Test did not show a statistically significant increase of value, and the impact of training was borderline moderate and high in:
- Shoulder Mobility (left shoulder) (SM॥) score from the initial $(M=2.733, S D=0.458)$ to the final measurement $(M=2.867, S D=0.352), \mathrm{t}(14)=$ $1.468, \mathrm{p}<0.164$ bidirectionally. The average decrease in $\mathrm{SM}_{\|}$was -0.391 , while the $95 \%$ confidence interval was in the range of -0.328 to 0.063 . The value of eta squared was $\varepsilon^{2}=0.142$.

- Shoulder Mobility (right shoulder) (SMrl) score from the initial $(M=2.733, S D=0.458)$ to the final measurement $(M=2.800, S D=0.414), \mathrm{t}(14)$ $=-1.468, \mathrm{p}<0.164$ bidirectionally. The average decrease in $\mathrm{SM}_{\mathrm{rl}}$ was -0.390 , while the $95 \%$ confidence interval was in the range of -0.328 to 0.062 . The value of eta squared was $\varepsilon^{2}=0.142$.

- Active Straight-Leg Raise (left leg) (ASLR\|) score from the initial $(M=2.467, S D=0.516)$ to the final measurement $(M=2.600, S D=0.507), \mathrm{t}(14)$ $=-1.468, \mathrm{p}<0.164$ bidirectionally. The average decrease in ASLR\| was -0.390 , while the $95 \%$ confidence interval was in the range of -0.328 to 0.062 . The value of eta squared was $\varepsilon^{2}=0.142$.

- Trunk Stability Push Up (TSP) score from the initial $(M=2.600, S D=0.507)$ to the final measurement $(M=2.733, S D=0.458), \mathrm{t}(14)=$ $1.468, \mathrm{p}<0.164$ bidirectionally. The average decrease in TSPU was -0.390 , while the $95 \%$ confidence interval was in the range of -0.328 to 0.062 . The value of eta squared was $\varepsilon^{2}=0.142$.

Handball has one of the highest injury rates among collective sports [44]. Studies have shown that large international handball tournaments have an incidence of 100 injuries per 1000 match hours [36, 44-45]. Injury incidence in young handball players has been reported to be 9.9 per 1000 match hours and 0.9 per 1000 training hours [46], which reduces with age. Young male handball players are at 1.8 greater risk of injury than their female peers [47]. Recent studies have shown that $23 \%$ of adolescent handball players in one season have had significant issues with their shoulder joints, half of which reported to have been completely unfit for competitive matches [48].

Most authors researching the matter agree that screening tests can be a tool to predict injuries [49-52]. Screening tests are a necessity in elite handball, especially in adolescent players, due to the high risk of injury [53]. One of the most commonly used movement screening test batteries for predicting risk of injury is the FMS ${ }^{\mathrm{TM}}[50,52]$.

The prospect research, using the $\mathrm{FMS}^{\mathrm{TM}}$ test battery, first diagnosed the quality of the participants' movements (initial state). Then, an exercise protocol 
for improving mobility and an exercise protocol for improving body stability were implemented over three months. The protocols were carried out twice a week in $\sim 10-15$-minute intervals. During this period, the players' injuries were recorded. At the end (final state), re-testing was done using the FMS ${ }^{\mathrm{TM}}$ test battery.

Analysis of the initial testing (Figure 3 ) shows that three players, or $20.0 \%$ (N. M.; I. M.; and M. B.) have an overall score of $\leq 14$, which indicates an increased risk of injury. Namely, prior studies have found that scores under 14 points in the $\mathrm{FMS}^{\mathrm{TM}}$ test battery are at 1.5 times [51], 2.7 times [50] and as much as 5 to 10 times higher risk in rugby [54], which, much like handball, carries a high risk of injury. Moreover, studies have shown that players with an overall score in the FMS ${ }^{\mathrm{TM}}$ test battery of $\leq 14$ points, in addition to getting injured more often, take longer to recover [55]. The remaining 12 players $(80.0 \%)$ have an overall score in the FMS ${ }^{\mathrm{TM}}$ test battery of $>15$, which indicates that this generation of young handball players of the RK Borac handball club has a developed quality of movements. Their scores range from 15 to 20 points. Of the three players whose overall score was below 14 points, two play in the pivot position. They are also the only two players whose BMIs indicate overweight (N.M. $=25.8 ;$ M.B. $=28.1$ ) in relation to the average $B M I$ of the whole team $(M=22.640, S D=$ 2.042). Considering their position and activity in offence and defence, and constant contact with opponents, a larger body mass, i.e. an increased BMI is to be expected. Research has confirmed that pivots are voluminous and carry slightly more fat tissue [5657]. Previous studies have found that there is a negative relation between BMI scores and $\mathrm{FMS}^{\mathrm{TM}}$ scores in the general population both in children and in adults [23, 58-59] as well as in NFL players, and the relation is especially prominent for lower extremities [60].

Three players (N.M.; B.M.; L.T.) were categorized as "asymmetric", since their ratio of asymmetry exceeded $15 \%$. The $15 \%$ limit was chosen because it can increase risk of sport injuries [61].

The participants had the lowest scores in the initial measurement in Rotary Stability Left $(M=1.867, S D=$ 0.516), and in tests: Rotary Stability Right $(M=2.000$, $S D=0.665)$, Deep Squat $(M=2.067, S D=0.704)$ and Hurdle Step Left $(M=2.067, S D=0.594)$. The participants had the highest scores in the initial measurements in tests: Shoulder Mobility, specifically
Right $(M=2.800, S D=0.414)$ and Left $(M=2.733$, $S D=0.458)$.

The Rotary Stability test evaluates the stability of the pelvis, torso and shoulder blades when performing a movement that involves moving upper and lower extremities. They require coordination, but also kinesthesia of the torso with the upper and lower extremities. When two extremities are lifted off the ground, the most prevalent component of rotation is the one that indicates that the rotation stability test evaluates trunk stability in the transversal plane. Three players exhibited a special deficit (A.G., M.B., K.Š.), and in both tests (Left and Right) they were graded with 1 . Based on the characteristics of the game of handball, "the body gains a rotational pattern during the unilateral movement, and the athlete must be able to maintain the stability" (p. 957) [37]. When it comes to young handball players, it is clear from this study that the obtained initial scores in Rotary Stability indicate an insufficient implementation of body stability exercises in the preparatory part of training sessions.

Low scores in the Deep Squat test belong to three players who were graded with 1 (I.M., M.B., L.T.). These three players had an increased risk of knee injury. The reasons of poor execution of the Deep Squat can be traced to poor mobility of the hip joint and the ankle joint, as a result of inadequate implementation of mobility exercises in the introductory part of the training sessions, or in other words, "the flexibility work usually limited to the hamstrings during training makes the athletes more disadvantaged in terms of flexibility of quadriceps and gastrosoleus muscle groups" (p. 957) [37]. Also, one of the players (M.B.) had a hip surgery some time ago. The knee is the most commonly injured body part in handball, and at the same time, knee injuries are the most severe in this sport [62]. Knee injuries are not the most common only in handball players under the age of 14 [63].

When jumping and shooting in handball, the lower extremities are in the air, so their stability heavily depends on their position on the ground [63]. The Hurdle Step test evaluates the ability to perform these movements. In our study, left leg tests have shown that the participants did not have a sufficient level in this test, especially two players (N.M., M.B). Considering the fact that $80 \%$ players are righthanded, meaning that their left leg is dominant in jumping, the engagement of its musculature may contribute to the shortening of the hamstring muscles (m.semitendinosus, m.semimembranosus i m.biceps 
femoris), and thus contribute to the decrease in its performance. Flexibility can be preserved and improved with the help of special training programs [64].

The young handball players had the highest scores in subtests Shoulder Mobility Left $(M=2.733$, $S D=0.458)$ and Right $(M=2.800, S D=0.414)$. The asymmetry rate in this test was low. Most athletes $(93 \%)$ use both their upper extremities equally. This indicates that these extremities were not subject to injury, which would have caused low values in this FMS $^{\mathrm{TM}}$ subtest. In any case, the results of the Shoulder Mobility subtest should be high in sports which primarily require the use of shoulders. Past injuries, improper treatment of the injuries and improper training cause lower scores in this subtest. Handball players employ different types of shots, with their forearm being above or below shoulder level, all the while exposing the shoulder joint to contact and blocks while the arm is extended upwards [65]. The forces exerted on the anatomical structures of the dominant shoulder while taking a shot can exceed the player's body mass 1.5 times [66-67]. This is why shoulder injuries are quite common in handball. The "handball shoulder" is a typical injury pattern that occurs due to excessive use, i.e. a large number of repetitions of shot movements using the dominant hand [68].

Exercise protocols for improving mobility and for improving body stability were then implemented. Their purpose was to improve the mobility of arms, shoulder girdles, thoracic vertebrae, hip and ankle, as well as the stability of the core, or the so-called abdominal ring, which is the basis for good performance of movements, not only in sports, but also in everyday activities. Also, the aim of performing the exercises bilaterally was to neutralize initial disbalance and to improve overall sports abilities.

It should be noted that in the observed period, between the two measurements, there were no player injuries.

After the second (final) measurement, the difference was $\varepsilon^{2}=2.152$, an indicator of impact magnitude for the $t$-tests. Since the value of eta squared was $>0.14$ [69], we can conclude that there is a high impact of the applied exercise protocols and that the difference obtained in between the two measurements indicates that there is a high impact of training on the functionality of the locomotor apparatus of young handball players.

When it comes to the scores of individual subtests of the $\mathrm{FMS}^{\mathrm{TM}}$ test battery in the final measurement, in
Figure 3 we can see that all the players scored $>14$. Except for two players who had the same score in the first and second measurements, all the other players scored better in the final than in the initial measurement. These two players had health issues, i.e. problems with their respiratory and cardiovascular systems, and due to medical examinations and required rest, during certain periods of the season they were exempt from training activities or underwent training of lower intensity. Quality of movement was significantly improved in three players whose overall scores in the initial measurement were $\leq 14$. Their BMIs in the second measurement indicated a mild growth $(M=22.907, S D=2.065)$ which is consequential to the increase of muscle mass due to focused daily training activity. Apart from the two pivots, whose BMIs scored as overweight in the initial measurement (N.M. $=26.0 ;$ M.B. $=27.8$ ), there is another player with a borderline score (L.B. $=25.2$ ). However, both pivots had significantly better overall scores in the FMS ${ }^{\mathrm{TM}}$ test battery, which means that BMI scores do not impact the quality of movements, but that they can be significantly improved through training.

The final scores indicated a high impact of training on the value of the Rotary Stability test (Left and Right), but without any statistical significance. It can be said that the results were expected, since the exercises which were directed at developing stability were carried out in an isometric mode, and as such could not have contributed to any statistically significant increase of dynamic stability, which is dominant in handball.

Results in Table 2 indicate that young handball players had a moderate to high impact of training, but have not displayed a statistically significant increase of the value of the following subtests of the FMS ${ }^{\text {TM }}$ test battery: Shoulder Mobility (left and right), Active Straight-Leg Raise (left leg) and Trunk Stability Push Up. A moderate to high improvement in these subtests induced by training activities can also be explained by the fact that the results of these tests were already satisfying already in the initial measurement, so there was no reason to expect that they will improve to the point where they would be statistically significant. Hence, in the Trunk Stability Push Up subtest, nine players $(60 \%)$ were graded with a 3 in the initial measurement (the rest were graded with a 2), while in the final measurement, two more players joined this group (73\%). 
Even though the FMS ${ }^{\mathrm{TM}}$ test battery was used extensively in the general population of adults, and not used often in the primary and secondary school population, there are a number of papers on the topic [70-72]. Results of recent studies show that $45 \%$ of boys aged 12-13 scored 14 or lower in the FMS ${ }^{\mathrm{TM}}$ test battery [73]. If we analyze the subtest scores, we can notice visible differences in Shoulder Mobility (Left and Right) and in Active Straight Leg Raise (Right). In these subtests, primary school pupils were in the lower mobility range [73]. Unlike for the general population, for young handball players it was in these tests that they had satisfactorily functional movement patterns.

The FMS TM test battery is attractive for researchers in sports, which is evident by the rising number of published papers on the topic for both recreation players [74] and top-level athletes [75]. Studies are being carried out in all levels both in professional [76] and semi-professional [77] sports, as well as in veteran [78] and adolescent [79] athletes.

When it comes to young athletes, a study in football showed that young football players: Achieved overall scores ranging from 9 to 16 points, where the majority did not have an overall score of $\leq 14$ points, almost half the players scored low (i.e. Individual score of <2) in the Deep Squat subtest, a majority performed poorly in the Trunk Stability Push-Up and Rotary Stability tests, and most of them had asymmetry in at least one of the five subtests [18]. Another study by the author of this paper, on young handball players, showed that they had better scores, because the overall scores increased from the 13-19 range in the initial measurement to the 15-21 range in the final measurement. Only $20 \%$ of the handball players had a total score of $<14$ in the initial, and none in the final measurement. However, just like in young football players, there were young handball players with low scores in the Rotary Stability subtest.

Engin Dinc et al. (2017) pointed out that they achieved success over four weeks of corrective exercises for young professional football players by using resistant rubber and a $65-\mathrm{cm}$ pilates ball, to correct deficiencies in the Deep Squat, In-Line Lunge and Trunk Stability Push-Up subtests, and other subtests as well [80]. Also, Mahsa Jafari et al. (2020) following their study on combat sports, concluded that "using [their] proposed training protocol, low FMS scores could be improved to 14 and higher" (p. 102) [81]. All this corroborates the results of this study, which indicate that the implemented exercises protocol over a specific period of time can improve the scores in the FMS $^{\mathrm{TM}}$ test battery overall, as well as in the individual subtests.

Prior research often discussed [25] whether some tests are more valid than others in terms of injury predictability. For example, in a study involving 31 players from youth handball teams from Bundesliga clubs, four players had contact injuries, and four had non-contact injuries. The FMS ${ }^{\mathrm{TM}}$ test battery scores did not show any difference between the injured and uninjured players. The same was true for the Front and Square Hop Tests, while there was a difference in the Y-balance Test and the Side Hop test. The authors of that study concluded that these two tests are especially suited for handball players [82]. Still, most other studies claim that athletes with dysfunctional fundamental movement patterns determined using the FMS $^{\mathrm{TM}}$ test battery have a higher chance of injury than those with high scores [22].

A drawback of this study is certainly the small participants sample $(n=15)$. But in literature, at least when this matter is concerned, there are quite a few studies done on a sample smaller than 30 participants. In any case, the study was done on a homogeneous, specific and high quality group of one team, which is limited to such a number of players. This is particularly important because of the intervention through an exercise protocol which was conducted uniformly.

\section{Conclusion}

Consequently, it can be concluded that the implemented eight-week specific protocol improved the functionality of the locomotor apparatus of young handball players. This is best evidenced by the fact that none of the players was injured during match time or training for the duration of the season in which the protocols were implemented, regardless of the scope and intensity of daily training sessions and focused match playing in all three competitions. It is certain that the FMSTM test battery is a significant pre-season tool for evaluating movement quality. Because of the large amount of information provided by the FMSTM, primarily on body posture, torso mobility and stability, and the asymmetry of specific body parts, this method surely deserves its place in working with athletes. In accordance with the obtained study results, exercise protocols for improving mobility and body stability are recommended to coaches, to be used in their daily work with athletes, especially in the preparatory period. 


\section{References}

[1] G. Cook, (2003) Athletic body in balance, Human Kinetics, Champaign, IL.

[2] K. Kiesel, L. Burton, \& G. Cook, Mobility screening for the core, Part 3: Implications for athletic low back pain, Athletic Therapy Today, 9(5) (2005) 38-41. [DOI]

[3] Wouter Welling, Effect of attentional focus on retention of drop jump landing strategies: Implications to enhance $\mathrm{ACL}$ injury prevention, MA Thesis, Center for Human Movement Sciences, University of Groningen (2014).

[4] C.W. Bond, J.C. Dorman, T.O. Odney, S.J. Roggenbuck, S.W. Young, \& T.A. Munce, Evaluation of the Functional Movement Screen and a Novel Basketball Test as an injury prediction tool for collegiate basketball players, Journal of Strength and Conditioning Research, 33(6) (2017) 1589-1600. [DOI] [PubMed]

[5] J. Gray, J.E. Taunton, D.C. McKenzie, D.B. Clement, J.P. McConkey, \& R.G. Davidson, A survey of injuries to the anterior cruciate ligament of the knee in female basketball players, International Journal of Sports Medicine, 6(6) (1985) 314-316. [DOI] [PubMed]

[6] T.E. Hewett, G.D. Myer \& K.R. Ford, Anterior cruciate ligament injuries in female athletes part 1, mechanisms and risk factors, American Journal of Sports Medicine, 34(2) (2006) 299-311. [DOI] [PubMed]

[7] P. Lisman, F.G. O'Connor, P.A. Deuster, \& J.J. Knapik, Functional movement screen and aerobic fitness predict injuries in military training, Medicine Science and Sports Exercise, 45(4) (2013) 636-643. [DOI] [PubMed]

[8] D.F. Murphy, D.A.J. Connolly, \& B.D. Beynnon, Risk factors for lower extremity injury: A review of the literature, British Journal of Sports Medicine, 37(1) (2003) 1329. [DOI] [PubMed]

[9] Eric A. Sorenson, Functional Movement Screen as a predictor of injury in High School basketball athletes. PhD Thesis, University of Oregon (2009)

[10] G. Cook, L. Burton, \& B. Hoogenboom, Preparticipation screening: the use of fundamental movements as an assessment of function part 1, North American Journal of Sports Physical Therapy, 1(3) (2006) 132139. [PubMed]

[11] G. Cook, L. Burton, \& B. Hoogenboom, Pre- participation screening: the use of fundamental movements as an assessment of function part 2, North American Journal of Sports Physical Therapy, 1(2) (2006) 62-72. [DOI]

[12] S.L. Zuckerman, A.M. Wegner, K.G. Roos, A. Djoko, T.P. Dompier, \& Z.Y. Kerr, Injuries sustained in National Collegiate Athletic Association men's and women's basketball, British Journal of Sports Medicine, 52(4) (2018) 261-268. [DOI] [PubMed]

[13] B.S. Dorrel, T. Long, S. Shaffer, \& G.D. Myer, Evaluation of the functional movement screen as an injury prediction tool among active adult populations a systematic review and meta-analysis, Sports Health, 7(6) (2015) 532-537. [DOI] [PubMed]

[14] K.D. Engquist, C.A. Smith, N.J. Chimera, \& M. Warren, Performance comparison of studentathletes and general college students on the functional movement screen and the $Y$ balance test, Journal of Strength and Conditioning Research, 29(8) (2015) 22962303. [DOI] [PubMed]

[15] D.M. Frost, T.A. Beach, J.P. Callaghan, \& S.M. McGill, Using the functional movement Screen to evaluate the effectiveness of training, Journal of Strength and Conditioning Research, 26(6) (2012) 1620-1630. [DOI] [PubMed]

[16] D.M. Frost, T.A. Beach, J.P. Callaghan, \& S.M. McGill, FMS scores change with performers' knowledge of the grading criteria - are general whole-body movement screens capturing "dysfunction?", Journal of Strength and Conditioning Research, 29(11) (2015) 3037-3044. [DOI] [PubMed]

[17] K. Kraus, E. Schütz, W.R. Taylor, \& R. Doyscher, Efficacy of the functional movement screen: A review, Journal of Strength and Conditioning Research, 28(12) (2014) 3571-3584. [DOI] [PubMed]

[18] V.B. Marques, T.M. Medeiros, F. DeSouza Stigger, F.Y. Nakamura, \& B.M. Baroni, The Functional Movement Screen (FMS) in elite young soccer players between 14 and 20: composite score, individual test scores and asymmetries, International Journal of Sports Physical Therapy, 12(6) (2017) 977-985. [DOI] [PubMed]

[19] G. Cook, (2011) Movement functional movement systems: Screening, assessment and corrective strategies. Lotus Publishing, Chichester, United Kingdom.

[20] G. Cook, L. Burton, B.J. Hoogenboom, \& M. Voight, Functional movement screening: The 
use of fundamental movements as an assessment of function part 1, International Journal of Sports Physical Therapy, 9(3) (2014) 396-409. [PubMed]

[21] K.C. Huxel Bliven, \& B.E. Anderson, Core stability training for injury prevention, Sports Health, 5(6) (2013) 514-522. [DOI] [PubMed]

[22] K. Kiesel, P.J. Plitsky, \& M.L. Voight, Can serious injury in professional football be predicted by a preseason Functional Movement Screen?, North American Journal of Sports Physical Therapy, 2(3) (2007) 147158. [PubMed]

[23] F.T. Perry, \& M.S. Koehle, Normative data for the Functional Movement Screen in middleaged adults, Journal of Strength and Conditioning Research, 27(2) (2013) 458462. [DOI] [PubMed]

[24] D. Džeko, \& M. Milanović, Functional assessment of movement, Kondicijski trening, 8(2) (2010) 23-27.

[25] R.J. Butler, M. Contreras, L.C. Burton, P.J. Plisky, A. Goode, \& K. Kiesel, Modifiable risk factors predict injuries in firefighters during training academies, Work, 46(1) (2013) 1117. [DOI] [PubMed]

[26] F.G. O'Connor, P.A. Deuster, J. Davis, C.G. Pappas, \& J.J. Knapik, Functional movement screening: Predicting injuries in officer candidates, Medicine Science and Sports Exercise, 43(12) (2011) 2224-2230. [DOI] [PubMed]

[27] M. Warren, C.A. Smith, \& N.J. Chimera, Association of the functional movement screen with injuries in Division I athletes, Journal of Sport Rehabilitation, 24(2) (2015) 163-170. [DOI] [PubMed]

[28] G. Cook, L. Burton, \& B. Hoogenboom, Functional movement screening: the use of fundamental movements as an assessment of function part 2, International Journal of Sports Physical Therapy, 9(4) (2014) 549563. [DOI] [PubMed]

[29] N. Vujkov, K. Idrizović, S. Vujkov, \& V. Panoutsakopoulos, Functionality of the movement in order to improve sports results and prevent injuries, Aktuelno u praksi, 24, (2013) 44-50.

[30] J. Bencke, D. Curtis, C. Krogshede, L.K. Jensen, T. Bandholm, \& M.K. Zebis, Biomechanical evaluation of the side-cutting manoeuvre associated with $\mathrm{ACL}$ injury in young female handball players, Knee Surgery Sports Traumatology Arthroscopy, 21(8) (2013) 1876-1881. [DOI] [PubMed]
[31] C. Karcher, \& M. Buchheit, On-court demands of elite handball, with special reference to playing positions, Sports Medicine, 44(6) (2014) 797-814. [DOI] [PubMed]

[32] S.S. Shojaedin, A. Letafatkar, M. Hadadnezhad, \& M.R. Dehkhoda, Relationship between functional movement screening score and history of injury and identifying the predictive value of the FMS for injury, International Journal of Injury Control and Safety Promotion, 21(4) (2014) 355-360. [DOI] [PubMed]

[33] R.H. Higashi, M.B. Santos, G.T.M. de Castro, B. Ejnisman, S.S. Sano, \& R.A. Da Cunha, Musculoskeletal injuries in young handball athletes: A cross-sectional study, Fisioter e Pesqui, 22(1) (2015) 84-89. [DOI]

[34] N. Giroto, L.C. Hespanhol Jr, M.R.C. Gomes, \& A.D. Lopes, Incidence and risk factors of injuries in Brazilian elite handball players: A prospective cohort study, Scandinavian Journal of Medicine Sciences and Sports, 27(2) (2017) 195-202. [DOI] [PubMed]

[35] N. Wedderkopp, M. Kaltoft, B. Lundgaard, M. Rosendahl, \& K. Froberg, Injuries in young female players in European team handball, Scandinavian Journal of Medicine Sciences and Sports, 7(6) (1997) 342-347. [DOI] [PubMed]

[36] T. Bere, J.M. Alonso, A. Wangensteen, A. Bakken, C. Eirale, H.P. Dijkstra, A. Hosny, B. Roald, \& N. Popovic, Injury and illness surveillance during the 24th Men's Handball World Championship 2015 in Qatar, British Journal of Sports Medicine, 49(17) (2015) 1151-1156. [DOI] [PubMed]

[37] E.S. Atalay, D. Tarakci, \& C. Algun, Are the functional movement analysis scores of handball players related to athletic parameters?, Journal of Exercise Rehabilitation, 14(6) (2018) 954-959. [DOI] [PubMed]

[38] G.B. Kürklü, A. Bayrak, M.P. Yargiç, \& N.ü. Yildirim, A comparison of functional movement abilities of elite male soccer and handball players, Turkish Journal of Sports Medicine, 54(3) (2019) 169-174. [DOI]

[39] R. Slodownik, A. Ogonowska-Slodownik, \& N. Morgulec-Adamowicz, Functional Movement ScreenTM and history of injury in the assessment of potential risk of injury among team handball players, Journal of Sports Medicine and Physical Fitness, 58(9) (2018) 1281-1286. [DOI] [PubMed]

[40] A. Letafatkar, M. Hadadnezhad, S. Shojaedin, \& E. Mohamadi, Relationship between 
functional movement screening score and history of injury, International Journal of Sports and Physical Therapy, 9(1) (2014) 2127. [PubMed]

[41] J.T. Monaco, \& B.J. Schoenfeld, A review of the current literature on the utility of the Functional Movement Screen as a screening tool to identify athletes' risk for injury, Strength and Conditioning Journal, 41(5), (2019), 17-23. [DOI]

[42] K.I. Minick, K.B. Kiesel, L. Burton, A. Taylor, P. Plisky, \& R.J. Butler, Interrater reliability of the functional movement screen, The Journal of Strength and Conditioning Research, 24(2), (2010), 479-486. [DOI] [PubMed]

[43] J.A. Onate, T. Dewey, R.O. Kollock, K.S. Thomas, B.L. Van Lunen, M. DeMaio, \& S.I. Ringleb, Real-time intersession and interrater reliability of the functional movement screen, The Journal of Strength and Conditioning Research, 26(2), (2012), 408-415. [DOI] [PubMed]

[44] A. Junge, G. Langevoort, A. Pipe, A. Peytavin, F. Wong, M. Mountjoy, G. Beltrami, R. Terrell, M. Holzgraefe, R. Charles, \& J. Dvorak, Injuries in Team Sport Tournaments during the 2004 Olympic Games, American Journal of Sports Medicine, 34(4), (2006), 565-576. [DOI] [PubMed]

[45] G. Langevoort, G. Myklebust, J. Dvorak, \& A. Junge, Handball injuries during major international tournaments, Scandinavian Journal of Medicine and Science in Sports, 17(4), (2007), 400-407. [DOI] [PubMed]

[46] O.E. Olsen, G. Myklebust, L. Engebretsen, \& R. Bahr, Injury pattern in youth team handball: A comparison of two prospective registration methods, Scandinavian Journal of Medicine and Science in Sports, 16(6), (2006), 426-432. [DOI] [PubMed]

[47] M. Moller, J. Attermann, G. Myklebust, \& N. Wedderkopp, Injury risk in Danish youth and senior elite handball using a new SMS text messages approach, British Journal of Sports Medicine, 46 (2012) 531-537. [DOI] [PubMed]

[48] M. Asker, L.W. Holm, H. Källberg, M. Waldén, \& E. Skillgate, Female adolescent elite handball players are more susceptible to shoulder problems than their male counterparts, Knee Surgery, Sports Traumatology, Arthroscopy, 26, (2018), 1892-1900. [DOI] [PubMed]

[49] R. Bahr, Why screening tests to predict injury do not work-and probably never will work: A critical review, British Journal of Sports
[50] N.A. Bonazza, D. Smuin, C.A. Onks, M.L. Silvis, \& A. Dhawan, Reliability, validity, and injury predictive value of the functional movement screen: A systematic review and meta-analysis, American Journal of Sports Medicine, 45(3), (2017), 725-732. [DOI] [PubMed]

[51] P.D. Bunn, A.I. Rodrigues, \& E. Bezerra da Silva, The association between the functional movement screen outcome and the incidence of musculoskeletal injuries: A systematic review with meta-analysis, Physical Therapy in Sport, 35, (2019), 146-158. [DOI] [PubMed]

[52] R.W. Moran, A.G. Schneiders, J. Mason, \& S.J. Sullivan, Do functional movement screen (FMS) composite scores predict subsequent injury? A systematic review with metaanalysis, British Journal of Sports Medicine, 51(23), (2017), 1661-1669. [DOI] [PubMed]

[53] J. Karlsson, A. Heijne, \& P. von Rosen, Handball and movement screening - can non-contact injuries be predicted in adolescent elite handball players? A 1-year prospective cohort study, Physiotherapy Theory and Practice, (2019), 1-7. [DOI] [PubMed]

[54] S.R. Duke, S.E. Martin, \& C.A. Gaul, Preseason functional movement screen predicts risk of time-loss injury in experienced male rugby union athletes, Journal of Strength \& Conditioning Research, 31(10) (2017) 2740-2747. [DOI] [PubMed]

[55] J. Tee, J. Klingbiel, R. Collins, M. Lambert, \& $Y$ Coopoo, Preseason Functional Movement Screen component tests predict severe contact injuries in professional Rugby Union players, Journal of Strength and Conditioning Research, 30(11) (2016) 3194-3203. [DOI] [PubMed]

[56] N. Foretić, O. Uljević, \& N. Rogulj, (2010). Anthropometric characteristics of handball players age 12 and 14, in M. Vantić-Tanjić (Ed.), Unapređenje kvalitete života djece $\mathrm{i}$ mladih (pp. 439-446). Tuzla, Bosnia and Herzegovina: Udruženje za podršku i kreativni razvoj djece i mladih.

[57] V. Srhoj, M. Marinović, \& N. Rogulj, Position specific morphological characteristics of toplevel male handball players, Collegium Antropologicum, 26(1) (2002) 219-227. [PubMed]

[58] M.J. Duncan, \& M. Stanley, Functional movement is negatively associated with weight status and positively associated with 
physical activity in British primary school children, Journal of Obesity, (2012) ID: 697563. [DOI] [PubMed]

[59] M.J. Duncan, M. Stanley, \& S. Leddington Wright, The association between functional movement and overweight and obesity in British primary school children, BMC Sports Science, Medicine and Rehabilitation, 5 (2013) 11. [DOI] [PubMed]

[60] C.P. Nicolozakes, D.K. Schneider, B.D. Roewer, J.R. Borchers, \& T.H. Hewett, Influence of body composition on Functional Movement Screen ${ }^{\mathrm{TM}}$ scores in college football players, Journal of Sport Rehabilitation, 27(5) (2018) 431-437. [DOI] [PubMed]

[61] J.J. Knapik, C.L. Bauman, B.H. Jones, J.M. Harris, \& L. Vaughan, Preseason strength and flexibility imbalances associated with athletic injuries in female collegiate athletes, American Journal of Sports Medicine, 19(1) (1991) 76-81. [DOI] [PubMed]

[62] B.L.S. Bedo, J.P.V. Manechini, M. Nunomura, R.P. Menezes, \& S.R.D. da Silva, Injury frequency in handball players: A descriptive study of injury pattern in São Paulo State regional teams, Motriz: Revista de Educação Física, 25(1) (2019) e101980. [DOI]

[63] P. Luig, \& T. Henke, (2011). Acute injuries in handball. In European Network for Sports Injury Prevention, poster presentation. Retrieved from http://www.safetyinsports.eu

[64] L.C. Decoster, J. Cleland, C. Altieri, \& P. Russell, The effects of hamstring stretching on range of motion: A systematic literature review, Journal of Orthopaedic and Sports Physical Therapy, 35(6) (2005) 377-387. [DOI] [PubMed]

[65] I. Bojić, Z. Valdevit, \& M. Veličković, The causes and prevention of injuries in handball, Teme, 46(2) (2020) 423-438. [DOI]

[66] B. Clarsen, R. Bahr, S.H. Andersson, R. Munk, \& G. Myklebust, Reduced glenohumeral rotation, external rotation weakness and scapular dyskinesis are risk factors for shoulder injuries among elite male handball players: a prospective cohort study, British Journal of Sports Medicine, 48(17) (2014) 1327-1333. [DOI] [PubMed]

[67] P. Lubiatowski, P. Kaczmarek, P. Cisowski, E. Breborowicz, M. Grygorowicz, M. Dzianach, T. Krupecki, L. Laver, \& L. Romanowski, Rotational glenohumeral adaptations are associated with shoulder pathology in professional male handball players, Knee Surgery, Sports Traumatology, Arthroscopy, 26(1) (2017) 67-75. [DOI] [PubMed]
[68] L. Laver, P. Landreau, R. Seil, \& N. Popović, (2018) Handball sports medicine: Basic science, injury management and return to sport, Springer-Verlag GmbH, DE Berlin, Germany.

[69] J.W. Cohen, (1988) Statistical power analysis for the behavioral sciences, 2nd ed, Lawrence Erlbaum Associates, Hillsdale, NJ.

[70] A. Abraham, R. Sannasi, \& R. Nair, Normative values for the Functional Movement ScreenTM in adolescent school aged children, International Journal of Sports Physical Therapy, 10(1) (2015) 29-36. [PubMed]

[71] B.E. Anderson, M.L. Neumann, \& K.C.H. Bliven, Functional movement screen differences between male and female secondary school athletes, The Journal of Strength \& Conditioning Research, 29(4) (2015) 1098-1106. [DOI] [PubMed]

[72] M.G. García-Jaén, S.S. Pérez, J.M. CortellTormo, A.F. Valero, \& R.C. Anta, Evaluation of fundamental movement patterns in children: Comparison of gender in primary school students, Retos: nuevas tendencias en educación física, deporte y recreación, 34 (2018) 282-286. [DOI]

[73] M. Vernetta-Santana, M. de Orbe-Moreno, E.M. Peláez-Barrios, \& J. López-Bedoya, Movement quality evaluation through the functional movement screen in 12- and 13year-old secondary-school adolescents, Journal of Human Sport and Exercise, 15(4) (2020) 918-931. [DOI]

[74] C. Agresta, M. Slobodinsky, \& C. Tucker, Functional movement screen: Normative values in healthy distance runners, International Journal of Sports Medicine, 35(14) (2014) 1203-1207. [DOI] [PubMed]

[75] Y. Li, X. Wang, X. Chen, \& B. Dai, Exploratory factor analysis of the functional movement screen in elite athletes, Journal of Sports Sciences, 33(11) (2015) 1166-1172. [DOI] [PubMed]

[76] D. Zalai, G. Panics, P. Bobak, I. Csáki, \& P. Hamar, Quality of functional movement patterns and injury examination in elite-level male professional football players, Acta Physiologica Hungarica, 102(1) (2015) 34-42. [DOI]

[77] P. Smith, \& M. Hanlon, Assessing the effectiveness of the functional movement screen $\left(\mathrm{FMS}^{\mathrm{TM}}\right)$ in predicting non-contact injury rates in soccer players, Journal of Strength and Conditioning Research, 31(12) (2017) 3327-3332. [DOI] [PubMed]

[78] D. Hammes, K. Aus der Fünten, M. Bizzini, \& 
T. Meyer, Injury prediction in veteran football players using the functional movement screenTM, Journal of Sports Sciences, 34(14) (2016) 1371-1379. [DOI] [PubMed]

[79] R.S. Lloyd, J.L. Oliver, J.M. Radnor, B.C. Rhodes, A.D. Faigenbaum, \& G.D. Myer, Relationships between functional movement screen scores, maturation and physical performance in young soccer players, Journal of Sports Sciences, 33(1) (2015) 11-19. [DOI] [PubMed]

[80] E. Dinc, B.K. Kilinc, M. Bulat, Y.T. Erten, \& B. Bayraktar, Effects of special exercise programs on functional movement screen scores and injury prevention in preprofessional young football players, Journal of Exercise Rehabilitation, 13(5) (2017) 535-540. [DOI] [PubMed]

[81] M. Jafari, V. Zolaktaf, \& G. Ghasemi, Functional Movement Screen composite scores in firefighters: Effects of corrective exercise training, Journal of Sport Rehabilitation, 29(1) (2020) 102-106. [DOI] [PubMed]

[82] C.H. Scheller, M. Keller, \& E. Kurz, Risk screening in handball: Which functional tests are useful?, Sportverletz Sportschaden, 32(3) (2018) 187-195. [DOI]

\section{Funding}

No funding was received to carry out this study.

\section{Authors Contribution}

Dr. Rađević - Conceptualization, Study design, Investigation, Data Collection, Resources; Dr. Simović - Formal Analysis, Methodology, Original Draft Preparation, Review and Editing; Dr. Ponorac Conceptualization, Methodology, Supervision; Dr. Drljača - Investigation, Data Collection, Original Draft Preparation.

\section{Ethics Approval}

Ethics approval was sought from Pan-European University Apeiron Banja Luka and Handball Club "Borac" Banja Luka.

\section{Informed Consent}

Written consent was obtained from the participants.

\section{Conflict of interest}

The Authors have no conflicts of interest to declare that they are relevant to the content of this article.
Does this article screened for similarity? Yes

\section{About The License}

(C) The Author(s) 2021. The text of this article is open access and licensed under a Creative Commons Attribution 4.0 International License 